\title{
Estudo preliminar sobre a qualidade de vida e sintomatologia depressiva de pacientes em fase pré e pós-transplante hepático
}

Preliminary study of quality of life and depressive symptomatology in pre- and post-transplant patients

Rosana Trindade Santos Rodrigues ${ }^{1,2}$, Wilze Laura Bruscato ${ }^{1,3}$, Ana Lúcia de Moraes Horta ${ }^{4}$, Luiz Antonio Nogueira-Martins ${ }^{5}$

\section{Resumo}

0 transplante hepático é um recurso caro e sofisticado e, quando o procedimento é bem sucedido, tende a proporcionar uma melhora física significativa, apesar da necessidade de acompanhamento médico e do uso de medicações imunossupressoras durante toda a vida. Uma das preocupações atuais das equipes transplantadoras é a qualidade de vida dos pacientes após o transplante, e a presença de sintomas depressivos de pacientes portadores de doença hepática crônica, transplantados e em lista de espera, para transplante de fígado. A amostra constou de 15 pacientes transplantados e 15 aguardando transplante. Foram utilizados: ficha sociodemográfica, Short-Form Health Survey (SF-36), Inventário Beck de Depresão (IBD) e a classificação de gravidade de doença Child-Turcotte-Pugh (CTP). A qualidade de vida dos transplantados apresentou melhor resultado quanto aos domínios do componente físico e pior nos domínios do componente mental do SF-36. Os resultados do IBD revelaram uma tendência dos transplantados a apresentar escores para depressão maior que aqueles em lista de espera. Os pacientes transplantados, embora com escores para depressão mais elevados, apresentaram melhora da capacidade física e da qualidade de vida em relação ao componente físico, porém mostraram piora em relação à condição psicossocial.

Palavras-Chave: Qualidade de vida; depressão; transplante de fígado.

\begin{abstract}
The liver transplant is an expensive and sophisticated resource and, when successful, provides a significant physical improvement, despite the need for medical surveillance and the use of immunosuppressive medications throughout life. One of the transplanting team's current concerns is the patients' quality of life after the transplant and the presence of depressive symptoms in transplanted patients with chronic liver disease and those in the waiting list for liver transplant. The sample consisted of 15 transplanted patients and 15 waiting transplant. The following were used to assess the patients: sociodemographic form, Short-Form Health Survey (SF-36), Beck Depression Inventory (BDI), and the Child-Turcotte-Pugh (CTP) rating of illness severity. The quality of life of those with a liver transplant produced better results concerning the SF-36 physical component areas and worst in the mental components. The BDI results revealed a tendency for those with a transplant to present greater depression scores than those in the waiting list. Even though the transplanted patients presented higher scores for depression, they showed an improvement in the physical capacity and quality of life in relation to the physical component, but showed worsening in relation to psychosocial condition.
\end{abstract}

Keywords: Quality of life; depression; liver transplantation.

Recebido: 22/11/2007

Revisado: $23 / 06 / 2008$

Aprovado: 02/07/2008

Serviço de Psicologia da Irmandade da Santa Casa de Misericórdia de São Paulo, São Paulo (SP), Brasil

2 Universidade Presbiteriana Mackenzie, São Paulo (SP), Brasil:

${ }^{3}$ Departamento de Psiquiatria e Psicologia Médica da Faculdade de Ciências Médicas da Santa Casa de São Paulo (FCMSCSP), São Paulo (SP), Brasil

${ }^{4}$ Departamento de Enfermagem da Universidade Federal de São Paulo (Unifesp), São Paulo (SP), Brasi

${ }^{5}$ Departamento de Psiquiatria e Psicologia Médica da Unifesp, São Paulo (SP), Brasil

Endereço para correspondência: Wilze Laura Bruscato, Rua Santa Isabel, 305, $7^{\circ}$ andar - Santa Cecília, CEP 01221-020 - São Paulo (SP)

Tel: (11) 3337-0905/3223-1321/Fax: (11)3354-1964,E-mail:wlbruscato@uol.com.br 


\section{Introdução}

O transplante hepático é um recurso da medicina dispendioso e sofisticado, e quando o procedimento cirúrgico é bem sucedido, proporciona melhora física significativa, apesar de o paciente necessitar de acompanhamento médico e de medicações imunossupressoras, para evitar a rejeição, durante toda a vida. Considerando todos os aspectos do tratamento, um dos fatores que preocupa as equipes transplantadoras é a qualidade de vida $(\mathrm{QV})$ destes pacientes.

No ano de 2006, o Brasil contava com 61 centros transplantadores, 45 deles ativos, incluindo-se o Programa de Transplante Hepático da Santa Casa de Misericórdia de São Paulo, local da coleta de dados do presente estudo. Em lista de espera para o transplante hepático estavam cadastrados no país, 6.288 pacientes, com tempo médio de espera de 72 meses e taxa de mortalidade na lista de $18,5 \%$. Em 2004, foram realizados 935 transplantes e, entre janeiro e junho de 2005, foram realizados $445^{1}$.

Ainda assim, os estudos realizados no país abordam a qualidade de vida apenas de portadores de doenças crônicas em geral ${ }^{2}$. Na literatura internacional, os vários estudos encontrados apontam para melhora da qualidade de vida desses pacientes, mas também para muitas dificuldades advindas das complicações clínicas e dos problemas emocionais adquiridos com o sofrimento na fase crônica da doença. Estes estudos também mostram que a depressão pode ter um papel importante na compreensão desses prejuízos da qualidade de vida ${ }^{3-13}$.

Os pacientes deste estudo estavam inseridos num programa de atendimento multidisciplinar, no qual recebiam tratamento clínico e cirúrgico, avaliação e acompanhamento social, nutricional e psicológico. $\mathrm{Na}$ prática clínica, verificava-se que estes pacientes apresentavam boa recuperação da sua capacidade física, mas também eram observados sintomas depressivos e dificuldades em retornar para suas atividades diárias habituais. A literatura ${ }^{4,7,9}$ refere que a gravidade da doença, por trazer experiências emocionais difíceis para o paciente, está intimamente associada à quadros de humor deprimido e à prejuízos na qualidade de vida, sendo comum que os sintomas depressivos dificultem a percepção de melhoras na qualidade de vida ${ }^{10}$.

Para os pesquisadores deste programa, a expectativa é de que o paciente em pós-transplante tenha melhor qualidade de vida (física e mental), menos sintomas depressivos e menor dependência física do que o paciente em pré-transplante. Assim, este estudo teve como objetivos avaliar e comparar a qualidade de vida, a prevalência de sintomas depressivos e o grau de dependência física de pacientes portadores de doença hepática crônica, transplantados e não transplantados de fígado, em lista de espera ${ }^{4,7,9}$

\section{Casuística e métodos}

Trata-se de um estudo descritivo de corte transversal, realizado no Ambulatório do Programa de Transplante Hepático da Santa Casa de Misericórdia de São Paulo.
A amostra constou de 30 pacientes adultos (idade mínima de 18 e máxima de 63 anos), dos quais 15 eram transplantados de fígado (no mínimo há dois meses e no máximo há 72 meses) e outros 15 , não transplantados, pareados por sexo e idade, em lista de espera do referido programa. O grupo de transplantados foi constituído por todos os pacientes transplantados no programa, que se encaixaram nos critérios de inclusão. Do grupo dos não transplantados, participaram pacientes escolhidos aleatoriamente, mas com busca de pareamento com o grupo de transplantados em sexo e idade e diagnóstico na lista de espera, desde que estivessem dentro dos critérios de inclusão. Os critérios de inclusão foram: pacientes de ambos os sexos, com idade igual ou superior a 15 anos e em abstinência alcoólica total. No grupo de transplantados, só foram incluídos pacientes não internados e que haviam recebido o órgão há pelo menos dois meses.

Foram utilizados, além da ficha sociodemográfica, um instrumento genérico de qualidade de vida, o The Medical Outcomes Study 36-item Short-Form Health Survey (SF-36) ${ }^{14}$, o Inventário Beck de Depressão $(\mathrm{IBD})^{15-17}$, traduzido e validado para o uso no Brasi ${ }^{18}$, que avalia sintomatologia depressiva. Este instrumento contém 21 itens, incluindo sintomas e atitudes, cuja intensidade varia de zero a três. Os itens se referem à tristeza, pessimismo, sensação de fracasso, sensação de culpa, sensação de punição, autodepreciação, auto-acusações, idéias suicidas, crises de choro, irritabilidade, retração social, indecisão, distorção da imagem corporal, inibição para o trabalho, distúrbio do sono, fadiga, perda do apetite, perda de peso, preocupação somática e diminuição da libido. Os escores indicativos de sintomatologia depressiva são: de dez a 18, sintomatologia depressiva de leve a moderada; de 19 a 29, sintomatologia depressiva de moderada a grave e de 30 a 63, sintomatologia depressiva grave. Para avaliar a gravidade da doença, utilizaram-se dados clínicos e laboratoriais da classificação Child-Turcotte-Pugh (CTP). Esta classificação está dividida em A, B e C ou numérica. Neste estudo utilizamos a divisão por letras que designa que A é a melhor condição clínica, B é a media e C a pior condição clínica do paciente.

Após a aprovação do projeto pelos Comitês Científico e de Ética, foi realizado o contato telefônico com os pacientes e agendados horários no ambulatório do Programa de Transplante Hepático. No dia e horário agendados, os pacientes foram informados pelas psicólogas pesquisadoras sobre o estudo e, após a assinatura do termo de consentimento livre e esclarecido, os pacientes responderam aos questionários. Os dados do CTP relativos à época do estudo foram coletados nos prontuários.

Para a análise estatística foi utilizado o banco de dados do Statistical Package for the Social Sciences (SPSS), versão 13.018. A análise estatística foi realizada em dois estágios com níveis crescentes de dificuldade. Inicialmente, através da estatística descritiva, foi realizada uma análise exploratória que forneceu a análise descritiva das freqüências. Em seguida, para responder às perguntas do estudo, verificar a homogeneidade dos grupos de pacientes nos domínios dos questionários relativamente às medidas obtidas foi utilizado o teste de Levene e avaliar se os pacientes transplantados diferiam dos não-transplantados 
quanto à qualidade de vida, foi realizado teste U de Mann-Whitney ${ }^{19}$. Análises univariadas foram realizadas na tentativa de verificar a existência de associação entre o questionário CTP, as escalas do SF-36 e os resultados do IBD. Foi utilizado o teste de ANOVA para se comparar em cada grupo o CTP (A vs B vs C). Para todos os testes estatísticos deste estudo, o nível de significância foi $\mathrm{p} \leq 0,05$.

\section{Resultados}

Oito pacientes $(53,3 \%)$ de cada amostra eram do gênero masculino e sete $(46,7 \%)$ eram do gênero feminino. A média de idade foi de 38,8 anos $(\mathrm{dp}=18,41)$ para os pacientes transplantados e de 45,2 anos $(\mathrm{dp}=14,97)$ para aqueles em lista de espera. A maioria dos pacientes em ambas as amostras (dez transplantados, 66,7\% e 11 não-transplantados, $73,3 \%)$ eram ou já tinham sido casados, e nove transplantados (60\%) e oito não-transplantados $(53,3 \%)$ não tinham companheiro(a). Quanto à etiologia dos pacientes transplantados, temos que: quatro pacientes (26,7\%) eram auto-imunes; quatro (26,7\%) tinham cirrose por vírus C; três $(20 \%)$ por vírus B; dois $(13,3 \%)$, cirrose por álcool e outros dois $(13,3 \%)$, cirrose por álcool mais vírus B e C, respectivamente. Daqueles em lista de espera, a etiologia se distribuiu da seguinte forma: um paciente $(6,7 \%)$ era auto-imune; sete $(46,7 \%)$ tinham cirrose por vírus C; três $(20 \%)$ por vírus B; dois (13,3\%) por cirrose por álcool e outros dois (13,3\%), cirrose por álcool mais vírus B. As características sociodemográficas e clínicas da população estudada são apresentadas na Tabela 1.

Com relação à qualidade de vida, os pacientes transplantados apresentaram escores maiores, estatisticamente significantes (melhor QV) nos domínios capacidade funcional $(\mathrm{p}=0,031)$ e estado geral de saúde $(p=0,015)$ do que os pacientes em lista de espera. Os pacientes que estavam em lista de espera apresentaram maiores escores estatisticamente significantes (melhor QV) nos domínios aspectos sociais $(\mathrm{p}=0,010) \mathrm{e}$ aspectos emocionais $(\mathrm{p}=0,019)$ do que os pacientes transplantados.

Tabela 1 - Variáveis sociodemográficas e clínicas dos pacientes transplantados e dos pacientes não transplantados

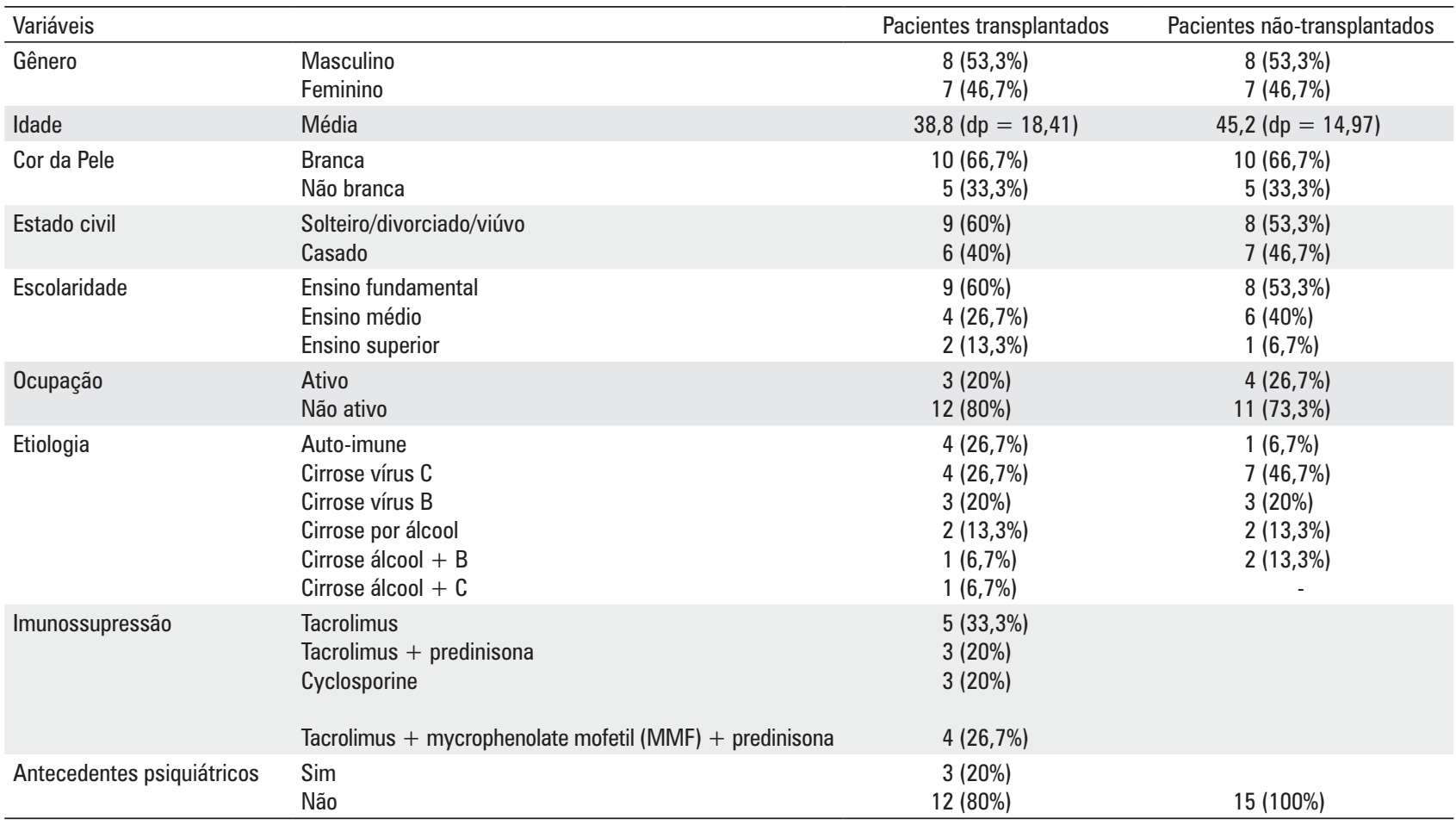

Tabela 2 - Valores descritivos e resultado da ANOVA para as escalas do SF-36 e para o IBD em cada categoria do CTP (A, B e C)

\begin{tabular}{|c|c|c|c|c|c|c|c|c|c|c|}
\hline \multirow{2}{*}{ Escalas } & \multicolumn{3}{|c|}{ CTP A $(n=10)$} & \multicolumn{3}{|c|}{ CTP B $(n=14)$} & \multicolumn{3}{|c|}{ CTP C $(n=6)$} & \multirow{2}{*}{$p$} \\
\hline & Média & \pm & $d p$ & Média & \pm & $\mathrm{dp}$ & Média & \pm & $\mathrm{dp}$ & \\
\hline Capacidade física & 71,50 & \pm & 20,44 & 64,57 & \pm & 31,94 & 83,50 & \pm & 14,14 & 0,336 \\
\hline Aspecto físico & 54,00 & \pm & 27,57 & 49,29 & \pm & 32,63 & 51,66 & \pm & 26,96 & 0,930 \\
\hline Dor & 85,60 & \pm & 18,91 & 61,40 & \pm & 22,29 & 90,63 & \pm & 20,00 & 0,007 \\
\hline Estado geral de saúde & 67,15 & \pm & 22,59 & 65,25 & \pm & 24,89 & 79,50 & \pm & 16,77 & 0,424 \\
\hline Vitalidade & 72,30 & \pm & 17,65 & 54,87 & \pm & 28,09 & 73,80 & \pm & 20,98 & 0,137 \\
\hline Aspecto social & 85,00 & \pm & 18,11 & 80,00 & \pm & 20,66 & 75,83 & \pm & 18,40 & 0,648 \\
\hline Aspecto emocional & 73,33 & \pm & 14,05 & 60,00 & \pm & 25,62 & 52,22 & \pm & 32,78 & 0,215 \\
\hline Saúde mental & 79,20 & \pm & 8,10 & 68,23 & \pm & 21,08 & 75,47 & \pm & 13,72 & 0,274 \\
\hline Inventário Beck de Depressão (IBD) & 6,80 & \pm & 2,57 & 10,86 & \pm & 8,42 & 11,17 & \pm & 8,73 & 0,334 \\
\hline
\end{tabular}


Os resultados do IBD mostraram que dois foram classificados como portadores de sintomatologia depressiva leve (escore entre dez e 18), três como sintomatologia depressiva moderada (escore entre 19 e 29) e um como sintomatologia depressiva grave (escore entre 30 e 63). Entre os pacientes não-transplantados, a média obtida (oito) não caracteriza quadro depressivo. A diferença entre os dois grupos, não foi estatisticamente significante $(p=0,786)$, embora tenha havido uma tendência dos pacientes transplantados a apresentarem os piores resultados quanto à sintomatologia depressiva.

Quando se utilizou a análise de variância (ANOVA) para testar a hipótese de que as várias médias obtidas por todos os pacientes (transplantados e não transplantados) nos domínios do SF-36 e no IBD eram iguais para as três categorias da avaliação pré-transplante, utilizada para medir a gravidade da doença (CTP), apenas o resultado do domínio dor do SF-36 variou com as categorias da classificação CTP de forma estatisticamente significante ( $p=0,007)$, de tal forma que, quanto maior a gravidade de doença, pior a qualidade de vida, com relação ao domínio dor. Já os demais questionários não apresentaram alteração estatisticamente significante quando comparados às categorias do CTP. Estes resultados estão na Tabela 2.

\section{Discussão}

Quando se comparou a qualidade de vida de pacientes transplantados e não-transplantados, verificou-se que o grupo de pacientes transplantados apresentou melhores índices de qualidade de vida em capacidade funcional e estado geral de saúde do questionário genérico de saúde (SF-36). Estes achados podem estar relacionados ao fato de que o transplante recupera a condição física dos pacientes, principalmente aqueles com diagnóstico de cirrose por vírus B e C - a maioria da amostra - que sofriam com limitações importantes, dada a gravidade da doença.

Os resultados deste estudo, no entanto, mostram que a recuperação da capacidade física não é elemento suficiente para a melhoria global da qualidade de vida dos pacientes transplantados. Nos domínios aspecto social, aspecto emocional e saúde mental, os pacientes transplantados apresentaram resultados piores que os não-transplantados, achados estes que corroboram estudos anteriores ${ }^{3,4,6,7,11-13}$.

Também no que diz respeito aos resultados da escala que avalia depressão, encontrou-se escores maiores no grupo de pacientes transplantados do que no grupo de não-transplantados. Porém, a diferença não foi estatisticamente significante.

Estes achados podem estar relacionados a dois aspectos. Primeiro, o fato de terem sido incluídos no estudo, pacientes transplantados há apenas dois meses. Este curto período pós-transplante, mesmo trazendo melhora do aspecto físico, pode ter influenciado os dados psicológicos. $\mathrm{O}$ segundo aspecto pode estar relacionado ao fato de que alguns pacientes, ao passar a fase inicial de excitação do pós-transplante, estavam retomando suas atividades, entrando em contato com sua real con- dição física, com os efeitos da imunossupressão, com a dependência dos serviços de saúde e com a incompleta recuperação, fatores que lhes indicavam que não reconquistariam a expectativa idealizada de uma vida normal. Como os pacientes passaram de hepatopatas para transplantados, continuaram na dependência dos serviços médicos e hospitalares, o que, na maioria das vezes, impedia uma rotina de vida normal. Estes dados corroboram estudos realizados recentemente ${ }^{13}$ que referem que a melhora imediata observada não é mantida ao longo do tempo.

A literatura da área expressa uma tendência otimista diante dos resultados da qualidade de vida após o transplante hepático. Neste estudo, constatou-se que a melhora está principalmente relacionada aos aspectos físicos, uma vez que há uma tendência a piora dos aspectos psicossociais. Embora nenhum paciente da amostra tenha tido recidiva da doença por vírus B ou C, este grupo teve uma vivência pré-transplante com sintomas graves da doença. Este fator, que pode ser considerado como elemento desestabilizador dos aspectos emocionais, pode ter interferido nos escores de qualidade de vida apresentados pelos pacientes transplantados que, de modo geral, estão abaixo do que seria considerado satisfatório. No entanto, ainda assim, em se tratando de aspectos físicos, os resultados são melhores do que na fase de espera pelo transplante. Quanto aos aspectos mentais, acredita-se que o prejuízo se dê, em sua maior parte, em razão da perda da expectativa idealizada de resgate da qualidade de vida, que possuíam antes da doença.

Os resultados obtidos neste estudo devem ser considerados com cautela, em função de outras limitações apresentadas. O fato de ter sido realizado em um único centro de transplante hepático, o tamanho da amostra e a não-utilização de um instrumento de avaliação de qualidade de vida específico para pacientes portadores de doenças hepáticas crônicas ou pacientes transplantados, são suas principais fragilidades. As justificativas para estes fatos estão relacionadas ao pequeno número de transplantes hepáticos realizados pelo centro e ao fato de que, na época do estudo, estava ainda em processo de validação, o primeiro instrumento de avaliação de qualidade de vida específico para este tipo de população. Assim sendo, optou-se pela utilização de um instrumento genérico.

Também deve ser considerada como uma limitação do estudo, o fato de não ter sido possível o pareamento absoluto dos grupos para a variável diagnóstico da doença hepática. Não havia número suficiente de pacientes em lista de espera neste centro que preenchessem os demais requisitos para este pareamento. Considera-se que esta variável pode apresentar influência nos resultados, uma vez que os sintomas diferem para cada diagnóstico. No entanto, embora não pareados em absoluto, os dois grupos foram compostos homogeneamente por pacientes com todos os diagnósticos (cirrose por hepatite auto-imune, cirrose por vírus $\mathrm{B}$ e C, cirrose por álcool, além das mistas, álcool e vírus).

Por outro lado, o estudo apresenta alguns aspectos que podem contribuir para o conhecimento científico na área da qualidade de vida dos pacientes portadores de doenças hepáticas crônicas e dos pacientes pós-transplante hepático, como o uso de um instrumento genérico de avaliação de qualidade de vida, associado a instrumentos que avaliam 
outros aspectos, como gravidade da doença e o surgimento de sintomatologia depressiva nos pacientes.

De qualquer forma, os aspectos aqui levantados merecem maior aprofundamento para que se possa traçar uma estratégia de melhor abordagem dos pacientes e da população para o transplante hepático. Pensou-se que uma proposta de intervenção interessante seria o investimento mais pontual na prevenção da depressão dos pacientes, enquanto aguardam o transplante. A partir deste estudo, os pacientes atendidos neste centro têm indicação de avaliação psiquiátrica e são tratados com antidepressivos, sempre que necessário, em conjunto com a psicoterapia.

Outras propostas seriam a criação de alternativas de seguimento pós-transplante que proporcionassem maior independência dos serviços hospitalares, para que o paciente consiga retomar suas atividades laborais, fator de extrema importância para a qualidade de vida, e a criação de um programa de reinserção social e profissional, através de um trabalho que implicasse na sensibilização da comunidade.
A qualidade de vida destes pacientes transplantados de fígado, conforme avaliada pelo SF-36, apresentou melhores resultados nos domínios do componente físico (capacidade funcional e estado geral de saúde). Quanto ao componente mental, houve piora nos domínios aspecto emocional e aspecto social.

A qualidade de vida destes pacientes não está relacionada à gravidade da doença avaliada na fase pré-transplante através da classificação do CTP.

Os pacientes transplantados apresentaram escores para depressão maior que os não-transplantados, em números absolutos, mas não houve significância estatística.

Sugere-se que outros estudos, inclusive em outros centros, sejam realizados para aprofundamento dos resultados aqui levantados, além de um estudo de seguimento desta amostra, uma vez que o número de estudos de seguimento com pacientes transplantados de fígado é pequeno, apesar de se tratar de um modelo adequado de pesquisa para avaliação da QV.

\section{Referências}

1. ABTO - Associação Brasileira de Transplante de Órgãos. Registro Brasileiro de Transplantes 2005;1:4-8.

2. Dantas RAS, Sawada NO, Malerbo MB. Pesquisas sobre qualidade de vida: revisão da produção científica das universidades públicas do Estado de São Paulo. Rev Latinoamer Enferm 2003;11(4):532-8.

3. Bravata DM, Olkin I, Barnato AE, Keeffe EB, Owens DK. Health-related quality of life after liver transplantation: a meta-analysis. Liver Transplantation Surg 1999;5(4):318-31.

4. Bona MD, Ponton P, Ermani M, lemmolo RM, Feltrin A, Boccagni P, et al. The impact of liver disease and medical complications on quality of life and psychological distress before and after liver transplantation. Journal Hepatol 2000;33(4):609-15.

5. Rodes J, Navasa M. Liver Transplantation and quality of life. Can J Gastroenterol 2000;14(8):693-9.

6. Ratcliffe J, Longworth L, Young T, Bryan S, Burroughs A, Buxton M; CostEffectiveness of Liver Transplantation Team. Assessing health-related quality of life pre and post-liver transplantation: a prospective multicenter study. Liver Transpl 2002;8(3):263-70.

7. Nickel R, Wunsch A, Egle UT, Lohse AW, Otto G. The relevance of anxiety, depression, and coping in patients after liver transplantation. Liver Transpl 2002;8(1):63-71.

8. Bucuvalas JC, Britto M. Health-related quality of life after liver transplantation: it's not all about the liver. J Pediatr Gastroenterol Nutr 2003:37(2):106-8.

9. O'Carroll RE, Couston M, Cossar J, Masterton G, Hayes PC. Psychological outcome and quality of life following liver transplantation: a prospective, national, single-center study. Liver Transpl 2003;9(7):712-20.
10. Fleck MPA, Lima AFBS, Louzada S, Schestasky G, Henriques A, Borges VR, Camey S, Grupo LIDO. Associação entre sintomas depressivos e funcionamento social em cuidados primários à saúde. Rev Saúde Pública 2002;36(4):431-8

11. Karan V, Castaing D, Danet C, Delvart V, Gasquet I, Adam R et al. Longitudinal prospective evaluation of quality of life in adult patients before and one year after liver transplantation. Liver Transpl 2003;9(7):703-11.

12. Sargent $S$, Wainwright SP. Quality of life following emergency liver transplantation for acute liver failure. Nurs Crit Care 2006;11(4):168-76.

13. Tome S, Wells JT, Said A, Lucey MR. Quality of life after liver transplantation. A systematic review. J Hepatol 2008;48(4):567-77.

14. Ware JE Jr, Sherbourne CD. The MOS 36-item short-form health survey (SF-36).I. Conceptual framework and item selection. Med Care 1992:30(6):473-81.

15. Beck AT, Ward CH, Mendelson M, Mock J, Erbaugh J. An inventory for measuring depression. Arch Gen Psychiatry 1961;4:561-71.

16. Beck AT, Steer RA, Garbin MG. Psychometric properties of the Beck Depression Inventory: twenty-five years of evaluation. Clin Psych Rev 1988:8:77-100.

17. Gorestein C, Andrade L. Inventário de Depressão de Beck: propriedades psicométricas da versão em português. Rev Psiquiatr Clin 1998;25(5): 245-50.

18. Norusis MJ. SPSS. Base 13.0. SPSS Inc. Chicago, 1999.

19. Conover WJ. Practical nonparametric statistics. 2nd ed. New York: John Wileyand Sons: 1980. 